International Journal of Computer Networks \& Communications (IJCNC) Vol.4, No.4, July 2012

\title{
Novel Cross-Layer Simulation Platform to INCLUDE REALISTIC CHANNEL MODELING IN SYSTEM SIMULATIONS
}

\author{
Getachew Redieteab ${ }^{1}$, Laurent Cariou ${ }^{1}$, Philippe Christin ${ }^{1}$, Jean-François \\ Hélard $^{2}$, and Nicolas Cocaign ${ }^{3}$ \\ ${ }^{1}$ France Télécom, Orange Labs, 4 rue du Clos Courtel, 35512 Cesson-Sévigné, France \\ \{getachew.redieteab, laurent.cariou, philippe.christin\} @orange.com \\ ${ }^{2}$ Université Européenne de Bretagne, INSA IETR UMR 6164, 35708 Rennes, France \\ jean-francois.helardeinsa-rennes.fr \\ ${ }^{3}$ NeoSoft France, 38 bis, rue de Rennes, 35510 Cesson-Sevigné, France \\ nicolas.cocaigneinge.neo-soft.fr
}

\begin{abstract}
Up to date wireless local access network (WLAN) simulation platform development efforts have concentrated either on the physical (PHY) layer or the medium access control (MAC) layer. The obtained performance is thus biased in that one layer has more weight than the other. On the other hand, an allinclusive simulator based on the actual platforms could be too much resource consuming. Simulator architectures are indeed tailor-made for one of the layers and thus not convenient for the other. That is why we propose a new IEEE 802.11n/ac multi-user simulation platform with reduced complexity. This platform is composed of an all inclusive PHY layer module and an elaborated MAC layer module working in a symbiotic manner. Both PHY and MAC layers being finely represented, an accurate modeling of reality is made possible. This PHY + MAC simulation platform can thus be an interesting tool for testing PHY-MAC cross-layer solutions for WLANs.
\end{abstract}

\section{KEYWORDS}

Cross-layer, hidden stations, IEEE 802.11n/ac, realistic channel modeling, simulation platform

\section{INTRODUCTION}

In the wireless local access network (WLAN) context, physical (PHY) layer or medium access control (MAC) layer performance evaluations can either use link-level or system-level simulations, depending on the layer to study in depth. Elaborated PHY layer simulators, notably including very fine channel models, consider at least one of the following assumptions if not all: full queues [1], oversimplified contention [2], or perfect channel state information feedback [3]. This kind of simulator is based on a "PHY-centric" study. Ptolemy II [4] and COSSAP [5] are such simulators. On the other hand, complex MAC layer simulators allow fine simulation of contention access and queue refilling, which is done according to application layer needs. But the PHY model these "MAC-centric" simulators use is oversimplified considering the complexity of the wireless channel. The latter is often modeled using either graph model $[6,7]$, ON/OFF model [8], or information-theoretic model [9]. Some models even use lookup tables (LUT) to enable simple link-to-system mapping technique (like packet error rate, PER, to signal-to-noise ratio, SNR, correspondence tables [10]). Sometimes more sophisticated techniques are used (bit error rate, BER, per block of subcarriers [11]). But in all cases the PHY layer has been simplified. NS3 [12] (or its previous version NS2 [10]) and Opnet [13] are such simulators. Therefore in both types of simulators, i.e. either PHY-centric or MAC-centric, one 
International Journal of Computer Networks \& Communications (IJCNC) Vol.4, No.4, July 2012

of the layers has been reduced in complexity, somewhat biasing the behavior of the global system.

However, building an all-inclusive and exhaustive simulator would be too complex of a solution. The specifications and characteristics of each layer being very different, a common ground might be more than difficult to find. All the while, the use of such simulators can be very beneficial, especially when studying phenomena requiring an accurate and realistic modeling of PHY and MAC layer mechanisms.

That is why we propose a new multiple user simulation platform composed of two parts working in a symbiotic manner. Each part finely characterizes the corresponding layer. The incorporation of an all inclusive IEEE 802.11n/ac [14, 15] PHY layer, containing a realistic channel model, and an elaborated IEEE 802.11n/ac MAC layer results in an accurate modeling of reality. PHY layer simulations are very time consuming because of channel tap computations. Consequently the simulation platform performs these simulations only when necessary. Such a knowledgeable mix of detailed PHY simulations and LUTs enables optimization with regards to time and computational resource consumption. In [16] a similar approach was used for 3GPP LTE-Advanced. Wi-Fi and cellular systems are quite different though. In Wi-Fi the access strategy is distributed and the data transmission channel is also used for signaling. An IEEE 802.11 specific simulator has to be used for Wi-Fi performance analysis studies. In [17] however, the authors have modeled both PHY and MAC layers of the IEEE 802.11a [18]. This simulator is very well done but does not comprehend either IEEE 802.11ac, or IEEE $802.11 \mathrm{n}$ enhancements, thus restricting its use to single antenna Wi-Fi standards.

We will firstly present the proposed simulator structure and detail the characteristics of the PHY chain, dynamic channel modeling, and MAC section. Then we will validate our simulation model through a well-known MAC functionality, data aggregation. A modified version of NS2 using LUTs serves as reference. Finally, the advantages of using such a model will be shown through a hidden node [19] scenario.

\section{Proposed Novel Simulator Structure}

\subsection{Presentation}

The principle that served as a basis for the elaboration of the simulator is the following: develop a MAC simulation module which interfaces with a PHY simulation module in a symbiotic manner. Indeed, in order to have a complete PHY-MAC simulator, it might be tempting to expand a PHY-exhaustive simulator to somehow faithfully comprehend the MAC layer, or the other way around. But this first sight solution implies a considerable increase in complexity. One of the layers' tailor-made simulator architecture has to be distorted so as to accommodate for the other layer. Therefore we propose to have two separate functions that interact only when necessary and exchange pre-processed information. We have an "OSI-like" structure, but with enhanced and completely dynamic interaction.

\subsubsection{PHY section with realistic channel modeling}

As implied before, we have used a fine grained PHY simulator as a basis of our cross-layer simulator. This custom Ptolemy II-like [4] PHY tool uses C++ environment, which eases functional block manipulation. It has allowed us to build IEEE 802.11n [14] and IEEE 802.11ac [15] compliant transmit (Tx) and receive (Rx) chains. TGn [20] and TGac [21] channel model blocks, which are used in the standardization process, represent the most substantial portion of the PHY section. These blocks enable a faithful representation of channel variations through time for IEEE 802.11n and IEEE 802.11ac systems (resp.) in a multiple-input, multiple-output (MIMO) context. 
International Journal of Computer Networks \& Communications (IJCNC) Vol.4, No.4, July 2012

\subsubsection{MAC section}

The NS3-like [12] MAC section deals with contention access, basic service set (BSS) management and control frame exchanges. Each frame is acknowledged if it is correctly received. The classical 802.11 contention access has also been extended to the IEEE 802.11e [22] quality of service (QoS) contention access and to the IEEE 802.11n packet aggregation. Multiple-user MIMO (MU-MIMO), as detailed in IEEE 802.11ac, is also implemented. In addition, rate adaptation algorithms, which are not specified in standards but are indispensable in any commercial product, are taken into account. It is also the case of MU-MIMO station selection algorithms, which will certainly be used in the IEEE 802.11ac standard. In short, we try to be as close as possible to realistic usage scenarios.

\subsubsection{Interactions between the PHY and MAC sections}

Once the transmitting station (STA) has been selected by the MAC section, information regarding the transmission (mainly MCS - Modulation and Coding Scheme - and data size) is handed over to the PHY section. The latter simulates transmission of information bits using the received parameters. Channel coding and modulation is done accordingly, followed by interaction with a fine grained channel, and a demodulation and decoding process to finish with. The PHY section then forwards the decoded bits, which will be checked through the included forward check sum (FCS), to the MAC section. Channel state information (CSI) can also be handed over, if necessary, to improve rate adaptation and MU-MIMO station selection. The two sections thus work sequentially and in an interdependent manner, so as to be sure to compass each layer's characteristics.

\subsection{Detailed PHY chain presentation}

\subsubsection{Global structure}

The most time and computational resource consuming block in WLAN link-level simulators is the channel block. This is due to channel tap generation and matrix manipulations. But a fine grained channel block is critical when modeling channel evolutions. Therefore for an intelligent use of resources, we have two PHY layers. One consists of a complete Tx chain, channel, and $\mathrm{Rx}$ chain. This model is used for the transmission of data frames. The precise effects (benefits as well as handicaps) of the physical medium and $\mathrm{Tx} / \mathrm{Rx}$ chains upon data transmission can thus be faithfully captured. The problem is that this process is time consuming. That is why we use another PHY model for control and management frames. This model is a simplified version of the first one. The success or failure of a frame is probabilistically determined based on LUTs (just like in some modified versions of NS2 and NS3). Control and management frames are normally sent using robust modulations. Therefore, compared to less robust $802.11 \mathrm{n}$ or 802.11 ac data frames, there is greater margin for correct reception. The particularities of the PHY chain have less impact on the outcome than for data frame reception.

The resulting optimized architecture will allow a simulation that is as close as possible to reality while minimizing global computational complexity.

\subsubsection{Parameters}

Our chain is designed so as to be compliant to $802.11 \mathrm{n}$ and $802.11 \mathrm{ac}$ standards. It is also shared by all STAs of the currently simulated scenario. Consequently $802.11 \mathrm{n}$ and $802.11 \mathrm{ac}$ parameters can be tuned during the scenario setup. In addition, all the transmission parameters (number of antennas, MCS, etc.) are dynamically reconfigurable according to the characteristics of the selected pair of STAs and the current transmission rate. This modular structure enables to compass a wider scope of scenarios. 
International Journal of Computer Networks \& Communications (IJCNC) Vol.4, No.4, July 2012

\subsubsection{Dynamic reconfiguration}

The PHY layer chain is common to all stations therefore it needs to be dynamically reconfigurable so as to meet the characteristics of each (e.g. number of antennas and data size). These are given over by the MAC section every time a STA has won access to the channel.

However, most PHY layer simulators (for e.g. Ptolemy II and COSSAP [5]) have functional blocks with static I/O FIFO sizes. The sizes of I/O FIFO buffers are set at the beginning of the simulation so as to fit a particular MCS. This is understandable considering that PHY layer simulations usually evaluate the performance of a single link, i.e. between a transmitter and a receiver. Having the same bridle in our fine grained PHY simulator while simulating multiple links, we built a "water pipe"-like (useful information + padding) structure for I/Os to workaround this flaw. At the beginning of the simulation, I/O FIFO sizes are set large enough so as to support the current simulation's maximum useful information size. However this bypass structure should not be much resource consuming because processing is only limited to the useful information, padding being neglected. Dynamic reconfiguration is enabled despite static I/O FIFO sizes.

\subsection{Dynamic channel modeling}

\subsubsection{Channel model}

As indicated before, one of the elements that renders our PHY layer simulation faithful to reality is the TGn [20] (or TGac [21]) channel modeling block. The latter is an SCM-like [23] geometric model based on stochastic modeling of scatterers, also called cluster model [24]. Fast fading and shadowing phenomena are also taken into account in the TGn and TGac models.

\subsubsection{Channel tap handling}

Channel taps characterize channel conditions between a pair of conversing STAs. The way they are handled determines the correspondence of the simulated scenario to reality. In addition, the channel modeling block being the most computation resource consuming element of the PHY layer chain, optimization can be done through wise channel management.

\subsubsection{Temporal}

Most PHY-centric simulations look after channel capacity. Therefore different channel conditions have to be considered so that the capacity may be ergodic, if possible. To do so, new channel taps are generated for every transmission. These taps are estimated by the receiver during the training sequence at the beginning of every frame. CSI is available at the receiver through this estimation process. On the other hand, CSI cannot be continually available at the transmitter for beamforming purposes, as assumed in classical simulators.

In the "real world", as in the proposed simulation platform, the transmitter has to send a sounding frame and wait for an estimate of channel taps (at the given time) to be fed back through a response frame. In addition, channel taps evolve through time (coherently to simulation time) while still remaining correlated. The fed back estimates can thus be used in following transmissions.

\subsubsection{Multiple station support}

Another advantage is facilitated support for multiple STAs. Channel taps between an oriented pair of STAs can be stored away so that the chain can be reused to simulate transmission between another pair of STAs, while having the possibility to recover, later on, the stored channel context. Space division multiple access (or MU-MIMO) is also rendered possible for 802.11ac implementation, by using stored taps to model crosstalk interference. 
International Journal of Computer Networks \& Communications (IJCNC) Vol.4, No.4, July 2012

\subsubsection{Gains compared to a MAC-centric approach}

We can see from what precedes that compared to a MAC-centric approach [6-12] which oversimplifies the PHY layer, our simulator structure, through realistic channel modeling and complete $\mathrm{Tx} / \mathrm{Rx}$ chains, allows a more reliable and more flexible PHY section.

\subsection{Detailed MAC section presentation}

\subsubsection{Global structure}

The MAC section contains the main MAC functions of the 802.11n (and 802.11ac) in conjunction with an application layer which generates packets. This is where the advantage of using $\mathrm{C}++$ programming language can be most clearly seen: we can generate as many applications per STA as desired, and create also as many STAs as necessary for the simulation thanks to the object concept in $\mathrm{C}++$. In the actual state of things, we have considered the infrastructure mode, i.e. with an access point (AP) assuming the management of the BSS. However, this can be easily extended to an ad hoc mode. In addition, we can also use our MAC section to generate multiple APs (operating on at least one common $20 \mathrm{MHz}$ channel) and see how the system reacts in an overlapping BSS context. Another advantage is that STAs supporting different bandwidths can also be associated to the same AP and one can easily study system behavior in such a scenario. We will note that NS2 and NS3 frameworks were used to develop this MAC section. Hence, this kind of structure offers a lot of possibilities for modeling different scenarios corresponding to every day use cases.

\subsubsection{Function presentation}

As indicated above, we can define as many applications as desired per node. The latter centralizes topology information (see Figure 1). Applications are managed by an application function which models higher layers and where the traffic category, rate, and duration are defined. Each application generates packets periodically, but with a random jitter for arrival fairness. The obtained traffic is then handed over to a network interface. The latter can either be AP specific or STA specific and handles traffic it relays to the contention and queueing function. In $802.11 \mathrm{n}$, this corresponds to the enhanced distributed contention access (EDCA). The access category (AC) having won access of the channel gives over its packet to the function handling data/acknowledgement transactions. Afterwards the PHY section takes over.

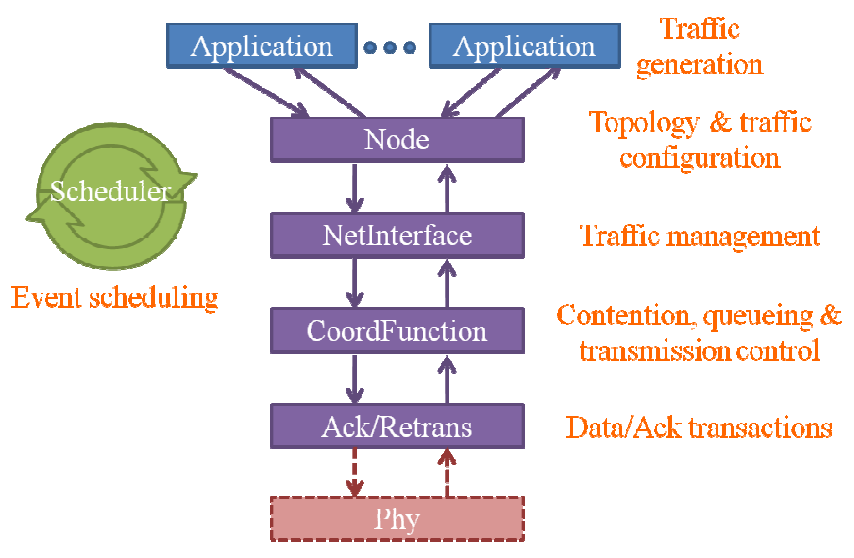

Figure 1. MAC section structure

We note that there is an event scheduler which takes care of the sequencing of channel access requests through callbacks. This way data, control, and management frames, and even collisions, are accounted for meticulously and in a timely manner. 
International Journal of Computer Networks \& Communications (IJCNC) Vol.4, No.4, July 2012

\subsubsection{Main MAC functions}

MAC particularities are considered mostly in NetInterface, CoordFunction and Ack/Retrans (as defined in Figure 1). Indeed, CSMA/CA (carrier sense multiple access with collision avoidance) with QoS, which is a schematic definition of EDCA, is an essential part of 802.11n. Every AC of every STA can contend for the channel and the outcome is decided in the CoordFunction function, which also builds the frames to transmit. Positive acknowledgment (ACK) is also a particularity of 802.11 systems. Ack/Retrans ensures that simple data MPDUs (MAC protocol data unit) or even aggregate MPDUs (A-MPDU) are correctly acknowledged. Still some MPDUs do not need acknowledgment but are essential in 802.11 systems. Beacons are among such frames. They are generated by the AP's NetInterfaces, which consider them as one of the many control, management or data flows that a NetInterface must manage.

\subsubsection{Gains compared to a PHY-centric approach}

As can be seen, traffic generation, queueing, and channel access, as well as acknowledgment, are all taken into account in this model. It would not have been the case in a PHY-centric approach [1-5] where all of the previous MAC and upper layer functions are simplified. The system behavior would diverge from reality. This is especially true in a multiple user context.

\section{SiMULATION SCENARIOS}

\subsection{Model validation through aggregation}

Before showing any of the improvements that this new simulation platform enables, the first thing to do is to validate the model. We propose to do this through a cut-and-dried concept of 802.11n systems, MPDU aggregation, using NS2 simulator with LUTs as reference.

\subsubsection{Reference structure: NS2 system simulator with LUT channel abstraction}

In the modified version of NS2 we have used, PHY layer performance is taken into account through LUTs, which are computed off-line through link-level simulations. This way the general particularities of the Tx chain, channel and Rx chain can be accounted for in a statistical manner. The success or failure of reception is established by randomly selecting a PER value and comparing it with the reference LUT PER value, for a given SNR. It will be our reference structure for the validation of the proposed simulation platform.

\subsubsection{Simulation parameters}

The TGn channel, IEEE 802.11n PHY, and IEEE 802.11n MAC simulation parameters (summarized in Figure 2) are the following:

- $1 \mathrm{AP}$ and 1 STA both having $2 \mathrm{Tx} / \mathrm{Rx}$ antennas (and supporting as many spatial streams), being placed $1 \mathrm{~m}$ apart;

- User datagram protocol (UDP) best effort traffic at $130 \mathrm{Mbps}$ for $8 \mathrm{~s}$. MAC service data units (MSDU) of 1500 octet (typical MAC payload format) sent using either simple MPDUs, or A-MPDUs of up to 2, 8, and 20 MPDUs depending on the simulation (tagged A-MPDU_2, A-MPDU_8, and A-MPDU_20 resp.);

- Adaptive multi rate retry (AMRR) rate adaptation algorithm [25] starting at minimum rate (i.e. $r_{0}=r_{1}=r_{2}=r_{3}=6.5 \mathrm{Mbps}, r_{0}$ being the highest rate) and used with rate counts (which are the numbers of retries per rate) set to $c_{0}=3, c_{1}=3, c_{2}=1$, and $c_{3}=3$; 
International Journal of Computer Networks \& Communications (IJCNC) Vol.4, No.4, July 2012

- Maximum transmit opportunity (TxOP) set to $3008 \mu \mathrm{s}$ (equivalent to standard maximum of video access category) to limit transmission duration to an end-usage-wise realistic TxOP;

- TGn channel model B (residential environment), with $20 \mathrm{MHz}$ bandwidth and central carrier frequency of $5.2 \mathrm{GHz}$ (channel $\mathrm{n}^{\circ} 40$ ). MCSs up to 15 are activated (because the use of two spatial streams is enabled);

- Tx power of $17 \mathrm{dBm}$ (half power), no antenna gains at Tx and Rx, Rx noise level of 7 $\mathrm{dB}$, and system loss of $8.5 \mathrm{~dB}$;

- 802.11n [14] standard compliant $\mathrm{Tx}$ and $\mathrm{Rx}$ chains using mandatory binary convolutional coding (BCC) and long guard interval (GI);

- Use same seed (50) to generate TGn channel taps for all simulations in this scenario. The rate adaptation algorithm can thus keep up with channel tap evolutions. These evolutions are coherent because the channel is initialized once.

The AP and STA are placed close to one another so that the rate adaptation algorithm can use the maximum PSDU (PHY service data unit) transmission rate (i.e. $130 \mathrm{Mbps}$ ). The CoordFunction can aggregate as many MPDUs as allowed by the maximum TxOP, using the current maximum rate (r0) to compute the frame's possible duration. We can thus send AMPDUs with a high number of aggregates (as much as 30). The proof of concept, where AMPDU_20s are to be used, is applicable. In addition, the chosen application rate corresponds to the maximum PSDU data rate. We thus insure that saturation could only be at MAC layer and/or PHY layer.

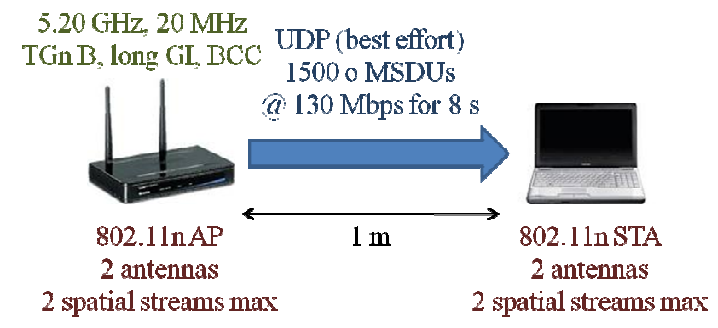

Figure 2. Simulation scenario and parameters for model validation

We will also note that all listed simulation parameters except those in the last two bullets, which are specific to the PHY section, are also used by the NS2 simulator. The two simulators are initialized with identical conditions.

\subsection{Contribution of the simulator in a hidden node scenario}

A STA establishes whether there is a signal through its carrier sense function. However if two or more STAs are out of range of each other but in range of another STA, carrier sense is eluded and collisions often occur. This is the hidden nodes problem [19]. The proposed simulator can be used to better characterize the consequences of this phenomenon.

\subsubsection{Classical structures}

\subsubsection{Reference structure: NS2 system simulator with LUT channel abstraction}

The NS2 (MAC-centric) simulator presented above can finely model contention access. However, if there is a collision detected by the event scheduler, colliding frames are 
International Journal of Computer Networks \& Communications (IJCNC) Vol.4, No.4, July 2012

automatically considered as corrupt, not acknowledged and must be retransmitted. This is done whatever the collision power, duration and location within the received frame. Clearly this way of doing things seems harsh. But considering that NS2 uses an abstracted PHY layer, it has no way of determining whether the collision leads to decoding errors or not. This simulator serves as our reference structure.

\subsubsection{Contribution of real-time channel modeling}

A PHY-centric simulator cannot, as such, model the complex channel access procedure of CSMA/CA. It cannot be used as a reference structure. However this approach can be interesting in that the interference caused by collisions can be simulated [26]. We can thus see whether the PHY layer Rx chain can recover from the induced signal distortions. The interference caused by the collision is considered as white Gaussian noise having the same power as the collision causing frame. It is added to the received signal on the concerned OFDM (orthogonal frequency division multiplexing) symbols. This can however be done only if the collision occurs in the PHY payload. If the PHY header is affected, we consider that the frame is lost. Indeed the PHY header contains synchronization, scaling, estimation, and payload MCS information. These fields enable the decoding of the PHY payload, and are thus crucial.

That is why in our simulation platform, in addition to collision-free data frames, the MAC section hands over data frames having undergone collision during PHY payload transmission to the fine grained PHY simulation chain. Information on the power of the collision inducing signal and on the relative position of collision affected OFDM symbols is also handed over.

\subsubsection{Simulation parameters}

Simulation parameters, depicted in Figure 3, are the same as in the previous scenario except concerning the following points:

- Two STAs placed diametrically with regards to the AP and transmitting UDP traffic to the latter;

- One of the STAs, STA1, moves further away from the AP (starting at $10 \mathrm{~m}$ ) in a periodic manner ( $5 \mathrm{~m}$ every $2 \mathrm{~s}$ ). The static STA, STA2, is placed far enough $(20 \mathrm{~m})$ so as to rapidly be in a hidden node situation with STA1;

- STA1 generates MSDUs of 100 octets and does not allow the use of MPDU aggregation. STA2 enables A-MPDU_2s;

- Seeds 50 and 52 are used for the TGn channels between STA1 and the AP, and STA2 and the AP (resp.).

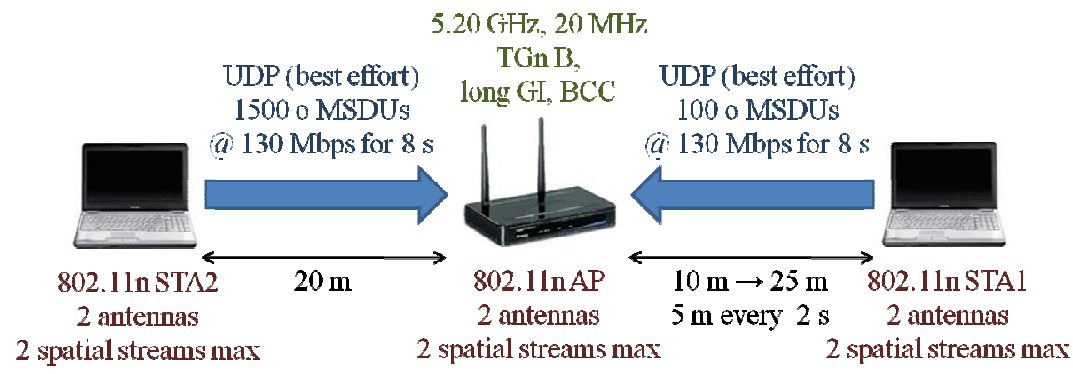

Figure 3. Simulation scenario and parameters exposing model contributions 
Here also, the new simulation parameters, except the ones in the last bullet, are also used in the NS2 reference simulator.

\section{Performance evaluation}

\subsection{Model validation through aggregation}

The UDP downlink (DL) rates obtained through the reference NS2 simulator and the new crosslayer simulator (denoted as XLS) for simple MPDU and A-MPDU_2 transmissions are illustrated in Figure 4. The reference NS2 simulator uses LUTs to decide whether a frame is correctly received or not. These correspondence tables are obtained by averaging PERs over at least 500 different channels for each SNR value. Particular evolutions of channel taps are thus smoothed out and a consistent PER-SNR curve is obtained for each MCS. XLS UDP DL curves are plotted for a specific channel (TGn with seed 50). They are thus much affected by the evolutions of this channel (fast fading, as well as slow fading). Still the obtained average UDP rates are the same for the two simulators, for simple MPDUs and A-MPDU_2s. In addition, by performing a sufficient number of simulations using different channels (thus different TGn seeds) and averaging, the results obtained by XLS should roughly match those of NS2 in this scenario.

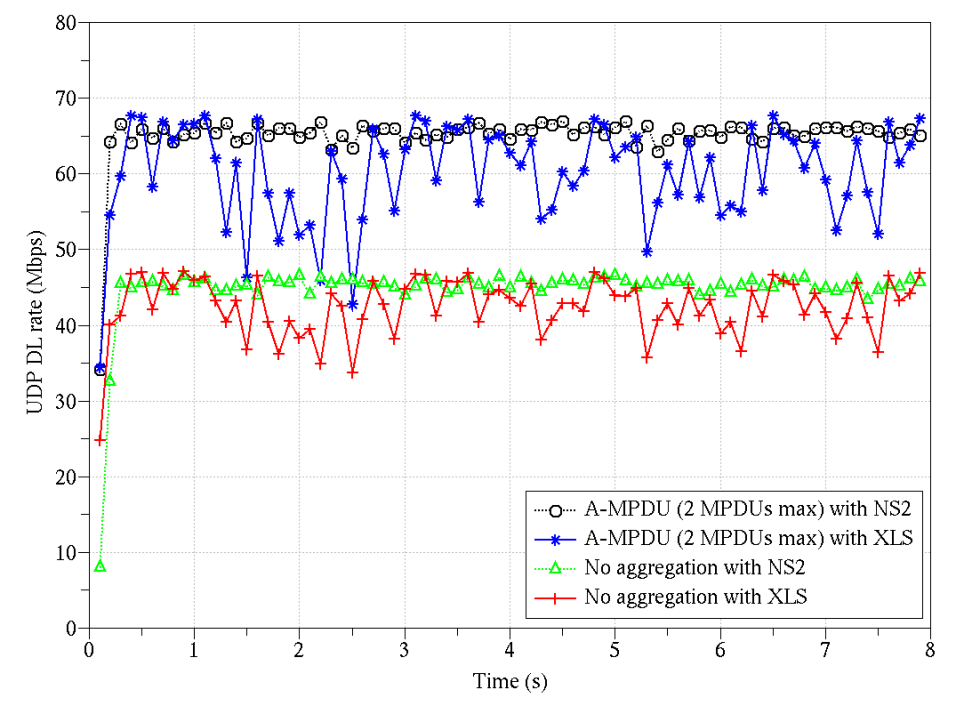

Figure 4. UDP downlink rates as a function of simulation time for transmissions without MPDU aggregation and with A-MPDUs (of at most 2 MPDUs) using the reference NS2 simulator and the proposed cross-layer simulator

An easy way to reach higher UDP rates, and see the interest of the new XLS platform, without modifying PHY parameters would be to use A-MPDUs with more MPDUs. The results of such simulations are given in Figure 5 (a), where A-MPDU_8s and A-MPDU_20s are used. When using the XLS, performance of A-MPDU_20 transmissions is even worse than that of simple MPDU transmissions (compare Figure 5 (a) and Figure 4). The latter have an average rate of 45 Mbps whereas the former have $40 \mathrm{Mbps}$.

Having designed the XLS platform to be as close as possible to real systems, channel taps are estimated at the beginning of the incoming frame. Rx channel estimation is simulated through the PHY header's long training frame sequences [22]. These estimates are then used for channel equalization of the rest of the frame. However if channel taps have changed notably in between, correct equalization cannot be done. The aging of these estimates can induce errors. There is nonetheless an amplifying factor: as indicated above, the number of MPDUs to be aggregated is 
International Journal of Computer Networks \& Communications (IJCNC) Vol.4, No.4, July 2012

determined using the highest rate $\mathrm{r} 0$ of the AMRR algorithm. For this rate and a maximum duration of $3008 \mu \mathrm{s}$, the TGn channel does not globally change much. But if consecutive errors induce rate decrease (through AMRR rate fallback), the frame will last longer. The odds of having important channel tap changes increase, eventually leading to errors. This chain reaction causes the UDP rate to drop notably. To verify this assertion, we have slightly modified the XLS PHY section so as to have OFDM symbol by OFDM symbol continuous estimation (XLS$\mathrm{CE}$ ). Estimates are updated throughout the whole frame reception process. The results obtained with this alteration are illustrated in Figure 5 (b). These results are on average similar to those obtained by NS2.

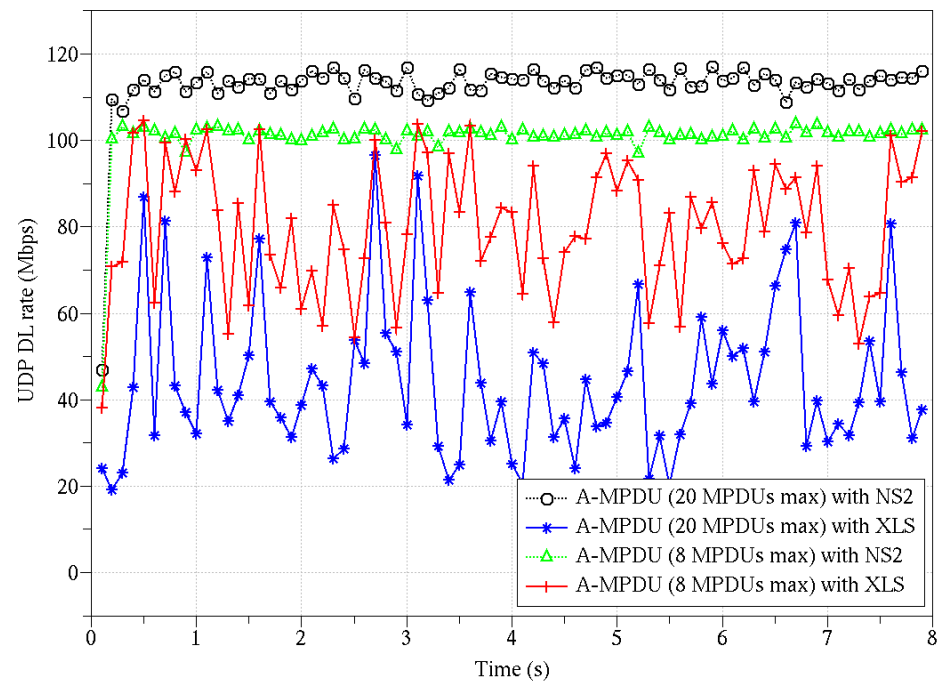

(a)

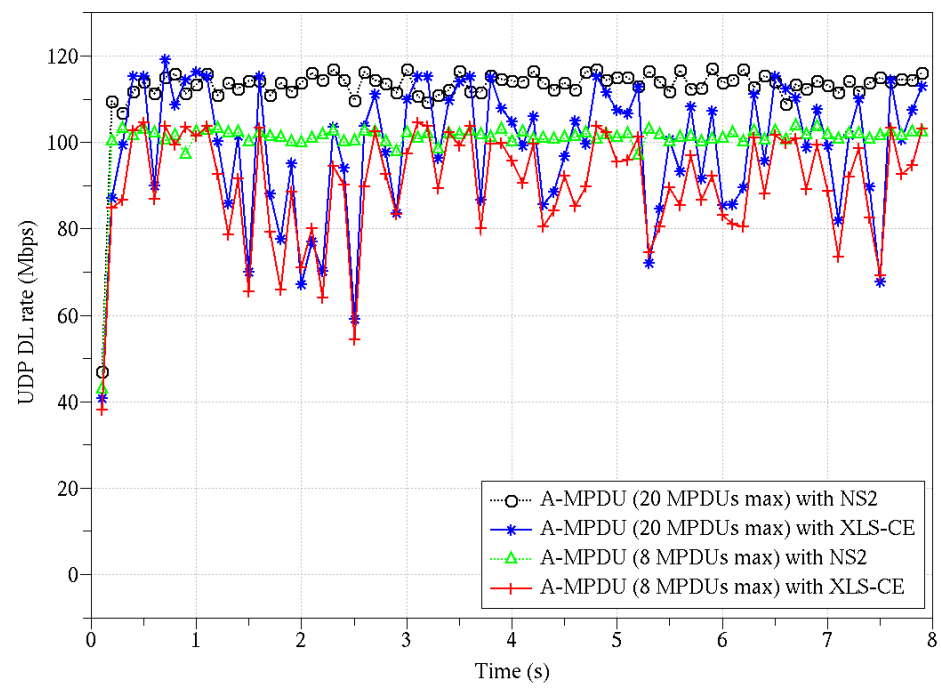

(b)

Figure 5. UDP downlink rates as a function of simulation time for transmissions with AMPDUs of at most 8 MPDUs and 20 MPDUs using NS2 and XLS. Channel estimation is done either once at the beginning of each frame (a), or for every OFDM symbol of each frame (b) 
Therefore the difference in UDL UL rates for A-MPDU_8 and A-MPDU_20 transmissions between NS2 and XLS is due to the aging of channel estimates. Taking into account this phenomenon can be a strong advantage in some studies. It is only with this kind of simulator that channel evolution (PHY) and MCS adaptation (MAC) can be simultaneously accounted for.

As stated above the effects of the rate adaptation algorithm on throughput are quite important. On all previous UDP DL graphs obtained using XLS, there is a rate decrease between $1 \mathrm{~s}$ and $1.5 \mathrm{~s}$. The evolution of PHY payload data rates is given in Figure 6 for this period. It can be seen that after $1.17 \mathrm{~s}$ the rate adaptation algorithm falls back to more robust modulations. The fallbacks could be due to ACK loss. As a control frame, the success or failure in receiving an ACK frame in XLS is determined through the use of LUTs. However, a uniformly distributed random variable is used for this purpose. Thus the odds of having bursts of error on ACKs only is very rare (if it were so PHY payload data rates of NS2 simulations, based also on LUTs, would have been less stable). The fallbacks could also be due to a deep fade of the TGn channel at that moment. These evolutions induce bursts of error. Knowing that the same channel (seed 50 ) is used for all the simulations of this scenario, the second assumption explains the rate decrease.

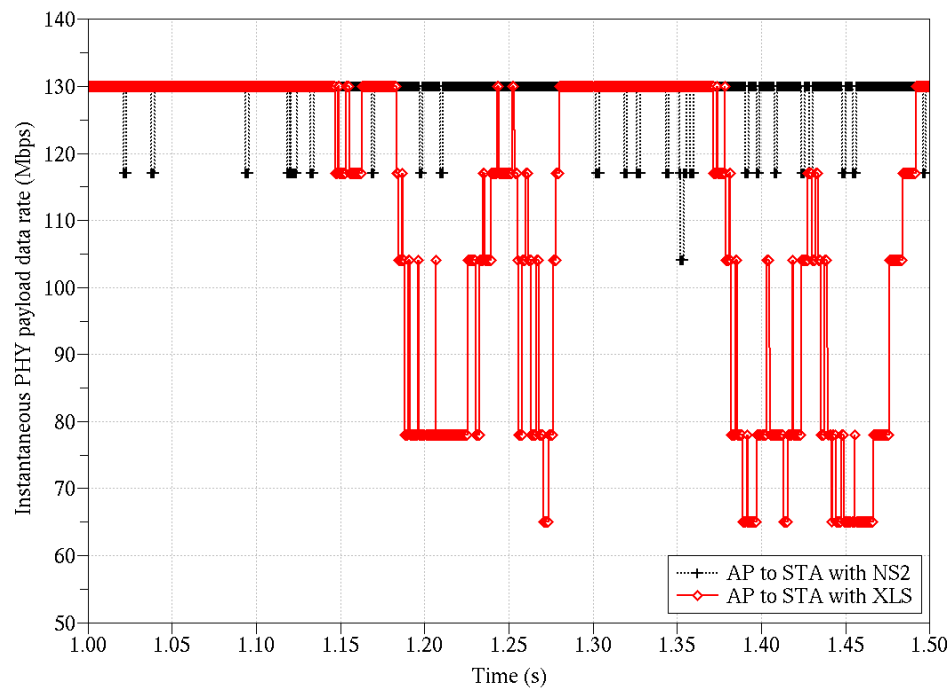

Figure 6. Instantaneous PHY payload data rates as a function of simulation time for A-MPDU transmissions of at most 2 MPDUs using NS2 and XLS

All the same, results show that the proposed model is valid. It can thus be used to fully model an 802.11n/ac environment. Some additional options like support for channel estimate aging can come in handy for finer analysis. Studies on the aging of CSI fed back to the transmitter, for beamforming purposes, can benefit from this XLS.

\subsection{Contribution of the simulator in a hidden node scenario}

In this scenario STA1 and STA2 are placed diametrically with regards to the AP. With STA1 moving away from the AP by $5 \mathrm{~m}$ every $2 \mathrm{~s}$ (see Figure 7 (a)), the consequent gap growing between STA1 and STA2 favors hidden node scenario. Indeed by using the minimum receiver sensitivity ( $-82 \mathrm{dBm}$ for BPSK $1 / 2$ modulations [14]) we can compute the range of each STA's transmission. Figure 7 (b) shows the maximum range covered by the AP, STA1 (with one ellipsoid per location), and STA2. 
International Journal of Computer Networks \& Communications (IJCNC) Vol.4, No.4, July 2012

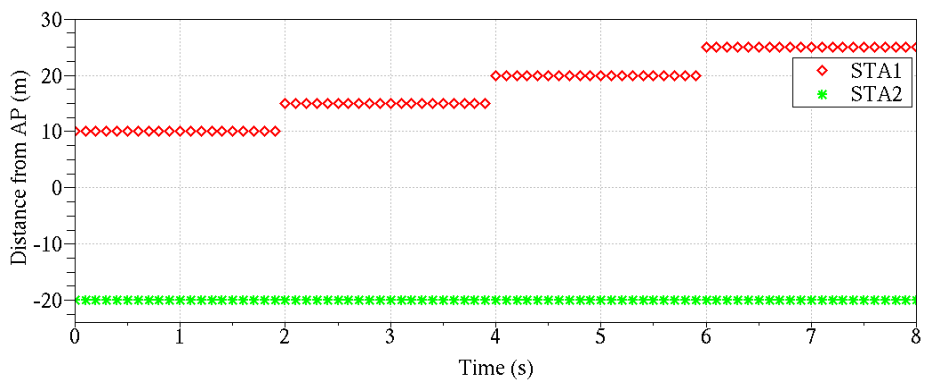

(a)

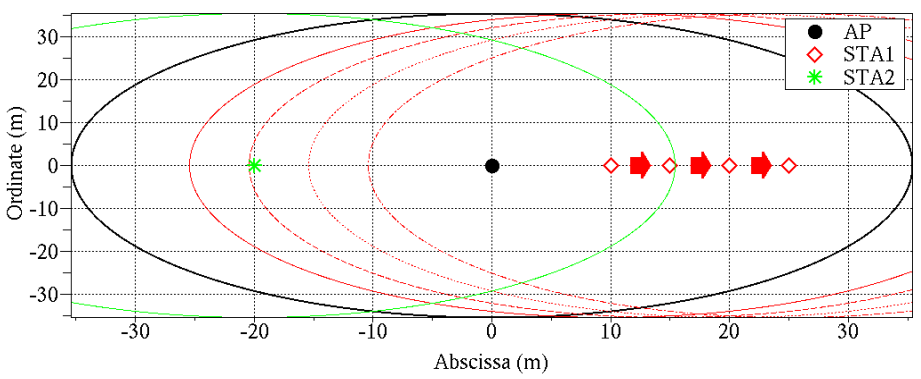

(b)

Figure 7. Positions of STA1 and STA2 through time (a) and on an AP centered grid displaying maximum range (b)

We can see from Figure 7 (b) that when STA1 is $10 \mathrm{~m}$ and $15 \mathrm{~m}$ away from the AP, the two STAs can sense each other. Collisions should thus be very rare. Because of CSMA/CA, there is collision only if both STAs have backoffs ending at the same time. In this case both STA's frames start at the same time and collision occurs during the PHY header. Both frames are regarded as lost, because of the importance of the PHY header. When STA1 is $20 \mathrm{~m}$ and $25 \mathrm{~m}$ away from the AP though, STA1 and STA2 become hidden nodes. Both STAs will transmit without taking the other into account, not being able to sense its transmissions. However ACKs coming from the AP are received by both. The occurrence and amplitude of collisions as perceived by the AP on frames from STA2 is given in Figure 8 (a). Figure 8 (b) illustrates the per-MPDU FCS error rate (FER) of frames received from STA2. The reader shall note that errors are detected by comparing the transmitted FCS and receiver computed FCS. The PSDU rates of ACK frames sent towards each STA are given, Figure 8 (c). By combining the three graphs we can see whether there was a collision (green rectangle in Figure 8 (a)) or not, if the received MDPU was correct (null FER, Figure 8 (b)) or not, and if the data frame has been acknowledged (strictly positive rate, Figure 8 (c)) or not. In addition all expected ACKs and block ACKs (BA) being registered, a "0 Mbps ACK rate" is equivalent to a missed ACK or a missed BA. 
International Journal of Computer Networks \& Communications (IJCNC) Vol.4, No.4, July 2012

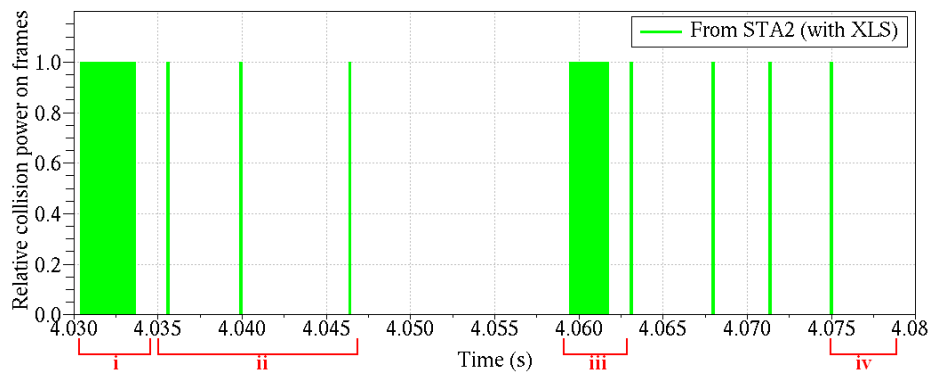

(a)

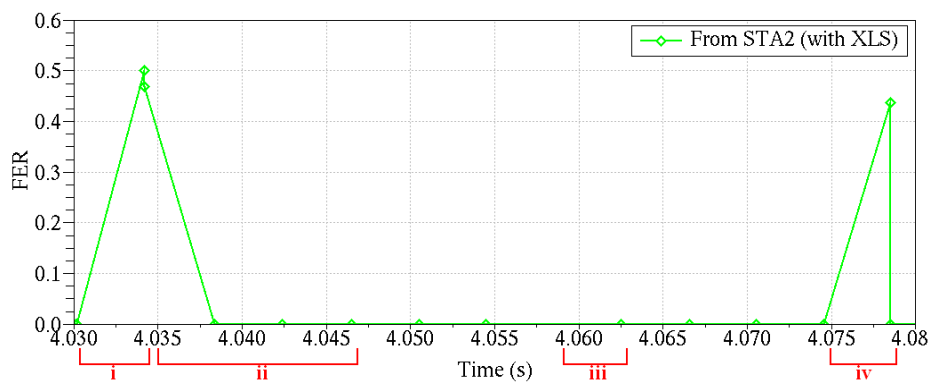

(b)

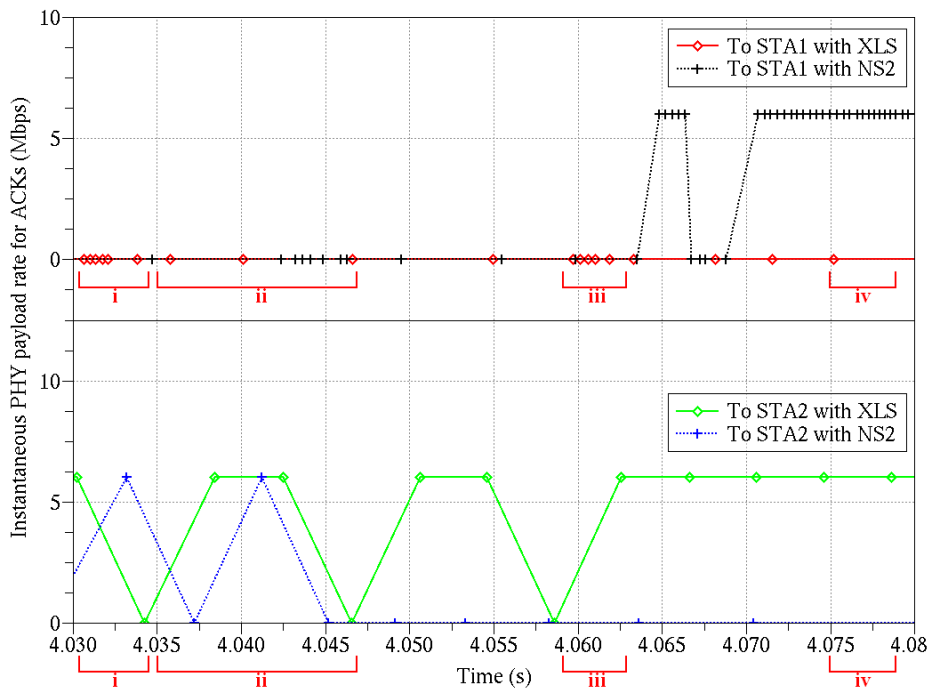

(c)

Figure 8. Forwarded collision amplitudes for STA2's transmissions (a), FER for frames received from STA2 (b), and ACK data rates used by the AP towards STA1 and STA2 (c)

During the first four illustrated milliseconds (interval i), STA1 has missed six ACKs, meaning that it has transmitted that many short (i.e. containing 100 octet data) frames to the AP. The latter has been receiving, in the same interval, a long (i.e. 2 MPDUs containing 1500 octet data each) frame from STA2. The PHY header of this frame being collision-free, its PHY payload is forwarded to the fine grained PHY section of the XLS. STA1's frames, received during STA2's PHY payload reception, have corrupted preambles and are thus not forwarded. Once all OFDM 
International Journal of Computer Networks \& Communications (IJCNC) Vol.4, No.4, July 2012

symbols of STA2's frame are received, the decoded bits are transferred to the MAC section which verifies whether the FCS is correct or not for each MPDU. It is based on the outcome of this test that the AP decides whether or not to acknowledge the concerned MPDU. FER is thus a concise indication of the impact of errors on each MPDU. FERs of MPDUs contained in an AMPDU are grouped on the graph. Using this information we can see that STA2's A-MPDU was lost due to collisions (FER of 0.5 and 0.47 for MPDU $n^{\circ} 1$ and $n^{\circ} 2$ resp.). The following three A-MPDU_2s sent by STA2 (interval ii) are correctly decoded (null FERs) despite collisions of important power. STA1 and STA2 are indeed at the same distance from the AP (i.e. $20 \mathrm{~m}$ ). However, only the first two frames are acknowledged, the BA for the last one having collided with a frame from STA1. Using the XLS platform thus enables to encompass the Rx chain's error correction capacity.

Another phenomenon concerning collisions in a hidden node scenario can be seen through the analysis of frames sent by STA2 at $4.059 \mathrm{~s}$ (interval iii) and $4.075 \mathrm{~s}$ (interval iv). Indeed the duration of the collision is not the only determining parameter, its localization within the frame is also very important. The first frame of the interval has a relatively long collision period. This is caused by the transmission of five frames by STA1, as implied by the corresponding missed ACKs. Still both its MPDUs are correctly acknowledged and the BA is correctly received. The second studied frame (interval iv) has a relatively short collision period. However the induced interference corrupted the first MPDU of this frame. Therefore the capacity of the Rx chain to recover from collisions complicates efforts to establish a simple collision threshold strategy. A simulator finely modeling both PHY (to compass the Rx chain) and MAC (to simulate the CSMA/CA driven access) layers is needed to study the effect of collisions.

Knowing that the PHY layer can correct a part of the collisions, the rather steady performance obtained for the total UDP UL rate (see Figure 9 (a)) with the XLS platform can be understood. NS2 considers that all collisions corrupt the frames they affect. Most STAs' frames are considered lost in the hidden node situation. Consequently the rate adaptation algorithm falls back and the CSMA/CA mechanism increases contention windows. The odds of having collisions decrease but the number of sent frames also decreases. This explains the important rate variations that we see for UDP UL rates for STA1 (see Figure 9 (b)) and STA2 (see Figure 9 (c)). When one of the STAs is penalized by the CSMA/CA mechanism for having had consecutive erroneous transmissions, the other STA makes use of this "collision-free" period to send its frames. The end result of NS2 simulations, in this particular scenario, diverges from the stable rate that would normally be expected. On the other hand, in XLS the AP manages to correct some of the collision induced errors, and the total UL rate remains stable. One will also notice that, with NS2, the UDP UL rate of STA2 falls after $2 \mathrm{~s}$. This is due to the fact that when STA1 is $15 \mathrm{~m}$ away from the AP, it is very close to the range limit of STA2 (see Figure 7 (b)). A small shadowing factor can topple things to a hidden node scenario, which is the case for NS2 simulation results.

Therefore there is clearly an advantage in having fine and real-time models of PHY and MAC layers when considering some phenomena.

\section{CONCLUSION}

In this paper, we presented a novel $802.11 \mathrm{n} / \mathrm{ac}$ simulation platform composed of a MAC simulation module which interfaces with a PHY simulation module in a symbiotic manner. This way, channel variations, $\mathrm{Tx} / \mathrm{Rx}$ chain specificities, and channel access mechanisms are faithfully taken into account, while minimizing computational resource consumption.

Through MPDU aggregation performance evaluation, we have validated our real-time channel modeling simulator in that similar performances are obtained as with a NS2 simulator using LUT channel abstraction. We have also shown in this paper that the proposed PHY/MAC 
International Journal of Computer Networks \& Communications (IJCNC) Vol.4, No.4, July 2012

simulation platform provides a more realistic modeling of some phenomena. The impact of channel estimate aging and the impact of deep fades can be accounted for while finely modeling contention access. In addition, the collision correction ability of the PHY chain was shown in a hidden nodes context.

The proposed $802.11 \mathrm{n} / \mathrm{ac}$ simulator structure is thus a very interesting platform for modeling phenomena and testing optimizations simultaneously involving PHY and MAC layers. MUMIMO performance evaluation studies can fully benefit from this platform.

\section{ACKNOWLEDGMENT}

The authors would like to thank Dr. A. Salah for his insightful comments and D. Morin for his help with NS2.

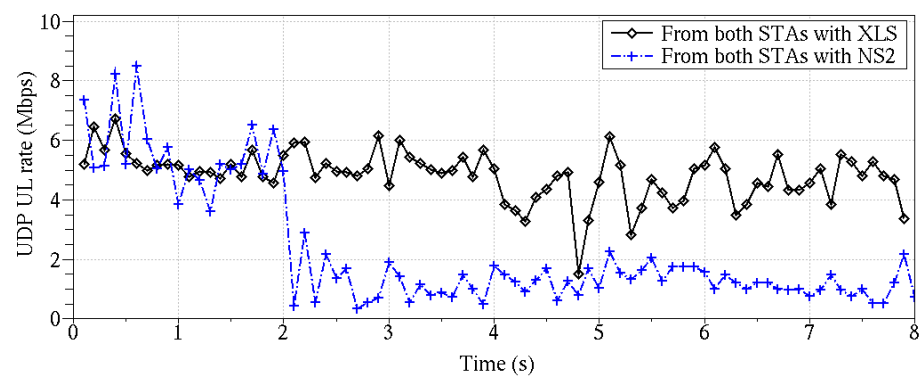

(a)

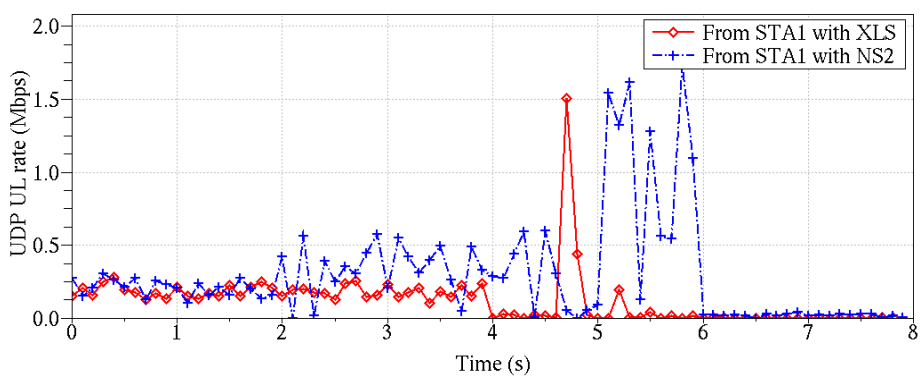

(b)

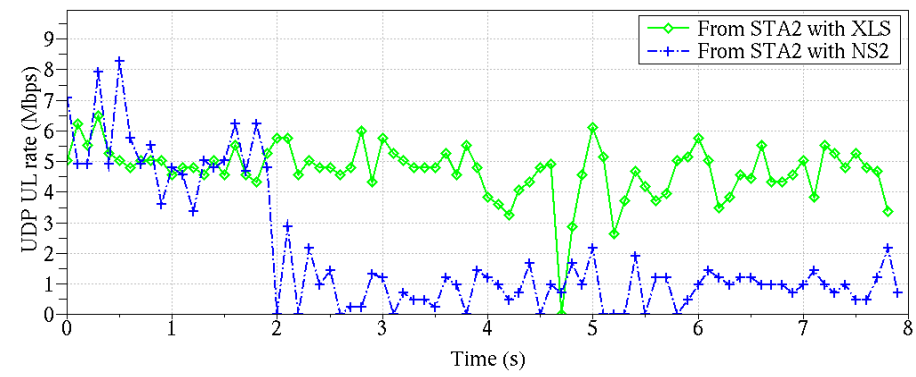

(c)

Figure 9. UDP UL as a function of simulation time for A-MPDU (of at most 2 MPDUs) transmissions using NS2 and the proposed cross-layer simulator for both STAs (a), for STA1 only (b), and for STA2 only (c) 
International Journal of Computer Networks \& Communications (IJCNC) Vol.4, No.4, July 2012

\section{REFERENCES}

[1] A. Goldsmith, S.A. Jafar, N. Jindal, and S. Vishwanath, "Capacity limits of MIMO channels," IEEE Journ. on Selected Areas in Comm., vol. 21, issue 5, pp. 684-702, Jun. 2003.

[2] H. Zheng, J. Wang, S. Zhou, and X. Su, "Minimal capacity loss round-robin scheduling algorithm for downlink beamforming multiuser MIMO systems," Int. Conf. on Wireless, Mobile and Multimedia Networks, pp. 1-4, Nov. 2006.

[3] Q.H. Spencer, C. B. Peel, A.L. Swindlehurst, and M. Haardt, "An introduction to the multi-user MIMO downlink,” IEEE Comm. Mag., vol. 42, issue 10, pp. 60-67, Oct. 2004.

[4] J. Buck, S. Ha, E.A. Lee, and D.G. Messerschmitt, "Ptolemy: A framework for simulating and prototyping heterogeneous systems," Int. Journ. of Comp. Simu., special issue on Simu. Soft. Dev., vol 4, pp. 155-182, Apr. 1994.

[5] G. Post, V. Zivojnovic, and S. Ritz, "Multiprocessor architecture extension for the blockdiagram-oriented tool COSSAP/DESCARTES,” Proc. Int. Conf. on Signal Processing Appl. and Techno., Oct. 1995.

[6] N. Kahale and P.E. Wright, "Dynamic global packet routing in wireless networks," Proc. of 16th Annual Joint Conf. of the IEEE Comp. and Comm. Soc., vol. 3, pp. 1414-1421, Apr. 1997.

[7] L. Qiao, K. Gyouhwan, and R. Negi, "Maximal scheduling in a hypergraph model for wireless networks,” IEEE Int. Conf. on Comm., pp. 3853-3857, May 2008.

[8] C.-P. Li and J.M. Neely, "Exploiting channel memory for multi-user wireless scheduling without channel measurement: Capacity regions and algorithms," Proc. of the 8th Int. Symp. on Modeling and Optim. in Mobile, Ad Hoc and Wireless Networks, pp. 50-59, Jun. 2010.

[9] K.N. Lau and Y.-K. Kwok, Channel adaptive technologies and cross layer designs for wireless systems with multiple antennas. Theory and application, Wiley, p. 428, 2006.

[10] N. Baldo, F. Maguolo, M. Miozzo, M. Rossi, and M. Zorzi, "ns2-MIRACLE: a modular framework for multi-technology and cross-layer support in network simulator 2," Proc. of the 2nd Int. Conf. on Perf. Eval. Method. and Tools, 2007.

[11] R. Srinivasan, J. Zhuang, L. Jalloul, R. Novak, and J. Park, "IEEE 802.16m evaluation methodology document," IEEE 802.16 Broadband Wireless Access Working Group, Jul. 2007.

[12] NS-3 IEEE 802.11 implementation. [Online]. http://www.nsnam.org/docs/release/3.12/models/singlehtml/index.html\#document-wifi

[13] OPNET Modeler Wireless Suite. [Online]. http://www.opnet.com/solutions/network_rd/simulation_model_library/wireless_lan.html

[14] IEEE Computer Society, "Wireless LAN medium access control (MAC) and physical layer (PHY) specifications: Enhancements for higher throughput,” IEEE Std 802.11n, Oct. 2009.

[15] $\quad 802.11$ Working Group, "Wireless LAN medium access control (MAC) and physical layer (PHY) specifications: Enhancements for very high throughput for operation in bands below 6GHz,” IEEE P802.11ac, Draft 1.0, May 2011.

[16] K. Kusume, et al., "System level performance of downlink MU-MIMO transmission for 3GPP LTE-advanced," IEEE Vehic. Techno. Conf., pp. 1-5, May 2010.

[17] J. Mittag, S. Papanastasiou, H. Hartenstein, and E. G. Ström, "Enabling accurate cross-layer PHY/MAC/NET simulation studies of vehicular communication networks," IEEE Proc., vol. 99, issue 9, pp. 1311-1326, Jul. 2011.

[18] IEEE Computer Society, "Wireless LAN medium access control (MAC) and physical layer (PHY) specifications: High-speed physical layer in the $5 \mathrm{GHz}$ band," IEEE Std 802.11a, Sep. 1999. 
International Journal of Computer Networks \& Communications (IJCNC) Vol.4, No.4, July 2012

[19] F.-Y. Hung and I. Marsic, "Access delay analysis of IEEE 802.11 DCF in the presence of hidden stations,” IEEE Global Telecom. Conf., pp. 2541-2545, Nov. 2007.

[20] V. Erceg, et al., “TGn channel models,” IEEE P802.11, May 2004.

[21] G. Breit, et al., "TGac channel model addendum,” IEEE P802.11, Mar. 2010.

[22] IEEE Computer Society, "Wireless LAN medium access control (MAC) and physical layer (PHY) specifications,” IEEE Std 802.11-REVma, 2006.

[23] D.S. Baum, J. Hansen, and J. Salo, "An interim channel model for beyond-3G systems: extending the 3GPP spatial channel model (SCM)", IEEE Vehic. Techno. Conf., vol. 5, pp. 3132-3137, Jun. 2005.

[24] A.A.M. Saleh and R. Valenzuela, "A statistical model for indoor multipath propagation,” IEEE Journ. on Selected Areas in Comm., vol. 5, issue 2, pp. 128-137, Feb. 1987.

[25] M. Lacage, M. Hossein, and T. Turletti, "IEEE 802.11 rate adaptation: A practical approach," MSWiM, Oct. 2004.

[26] G. Redieteab, L. Cariou, P. Christin, and J.-F. Hélard, "Cross-layer multichannel aggregation for future WLAN systems,” IEEE Int. Conf. on Comm. Sys., pp. 740-746, Nov. 2010.

\section{Authors}

Getachew Redieteab received the M.S. degree in electrical engineering from the National Institute of Applied Science (INSA) in Rennes, France, in 2009. He is currently working towards the Ph.D. degree in electronics and telecommunications within Orange Labs in Rennes, France Telecom Group, France. His research interests include PHY/MAC cross-layer design and optimization for wireless networks, accurate simulation environments, and multiple-user MIMO.

Dr. Laurent Cariou received his engineer diploma and his Ph.D in electronics and signal processing from the National Institute of Applied Sciences (INSA) in Rennes, France, in 2003 and 2006, respectively. Since then, he has been working as research engineer at Orange Labs in Rennes, France, where he conducted and supervised research on cross layer optimizations especially for WLAN systems. He is involved in several European and national research projects in the fields of mobile radio communications and has been representing Orange in the IEEE 802.11

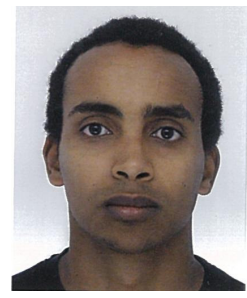
standardization group that defines physical and MAC layer of Wi-Fi technologies. He has been particularly active in $802.11 \mathrm{ac}, 11 \mathrm{ad}, 11 \mathrm{af}$ and $11 \mathrm{ah}$ working groups.

Philippe Christin holds an engineer degree from ENSAM (France) in 1993 and a M.S. degree in networking from Supelec (France) in 1994. He passed the Cisco certification program CCIP (Cisco Certified Internet Professional). Since 2007, Philippe Christin is a network engineer in Orange Labs in Rennes, France. He is participating in standardization working group IEEE 802.11ac (Very High Throughput below 6GHz) and IEEE P1905.1 (Convergent Digital Home Network). He is a technical expert in layer 2 technologies and layer 3 protocols. As part of his work, Philippe Christin has several patents filed in the field of

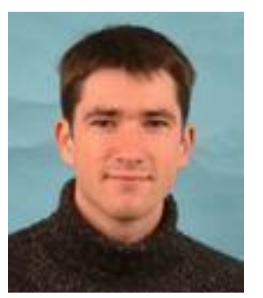
wireless communications, has an active contribution to collaborative European projects and is mentoring Ph.D students in WLAN Cross-Layer and Home networking areas. 
International Journal of Computer Networks \& Communications (IJCNC) Vol.4, No.4, July 2012

Pr. Jean-François Hélard received his Dipl.-Ing. and his Ph.D in electronics and signal processing from the National Institute of Applied Sciences (INSA) in Rennes, France, in 1981 and 1992, respectively. From 1982 to 1997 , he was research engineer and then head of channel coding for the digital broadcasting research group at France Telecom Research Center in Rennes. In 1997, he joined INSA, where he is currently Professor and Director of Research of the Institute. He is also Deputy Director of the Rennes Institute for Electronics and

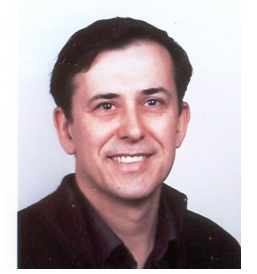
Telecommunications (IETR), created in 2002 in association with the CNRS. His research interests lie in signal processing techniques for digital communications, such as space-time and channel coding, multi-carrier modulation, as well as spread-spectrum and multi-user communications. He is involved in several European and national research projects in the fields of digital video terrestrial broadcasting, mobile radio communications and cellular networks, power-line and ultra-wide-band communications, cooperative communications and relaying techniques. Prof. J-F. Hélard is a senior member of IEEE, author and co-author of more than 200 technical papers in international scientific journals and conferences, and holds 14 European patents.

Nicolas Cocaign is working towards the M.S. degree in computer science from the Conservatoire National des Arts et Métiers (CNAM) of Paris, France. From 2000 to 2007, he worked in several domains such as realtime 2D/3D graphics and image processing. Since 2007, he has been working at NeoSoft as a network specialist engineer. He has studied and developed IEEE $802.11 \mathrm{a} / \mathrm{b} / \mathrm{g} / \mathrm{n}$ PHY layer and MAC layer simulators, with cross-layer design, for Orange Labs Rennes, France Telecom Group, France. His research interests include advanced

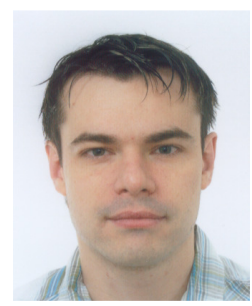
software architecture and cross-layer design. 